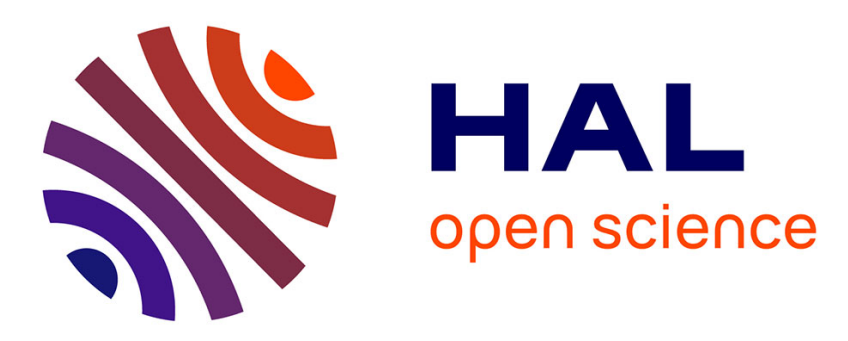

\title{
Intraoperative Resting State Functional Connectivity Based on RGB Imaging
}

Charly Caredda, Laurent Mahieu-Williame, Raphaël Sablong, Michaël Sdika, Fabien C Schneider, Jacques Guyotat, Bruno Montcel

\section{- To cite this version:}

Charly Caredda, Laurent Mahieu-Williame, Raphaël Sablong, Michaël Sdika, Fabien C Schneider, et al.. Intraoperative Resting State Functional Connectivity Based on RGB Imaging. European Conferences on Biomedical Optics, Jun 2021, Munchen (virtual), Germany. hal-03262003

\section{HAL Id: hal-03262003 https://hal.science/hal-03262003}

Submitted on 16 Jun 2021

HAL is a multi-disciplinary open access archive for the deposit and dissemination of scientific research documents, whether they are published or not. The documents may come from teaching and research institutions in France or abroad, or from public or private research centers.
L'archive ouverte pluridisciplinaire HAL, est destinée au dépôt et à la diffusion de documents scientifiques de niveau recherche, publiés ou non, émanant des établissements d'enseignement et de recherche français ou étrangers, des laboratoires publics ou privés. 


\title{
Intraoperative Resting State Functional Connectivity Based on RGB Imaging
}

\author{
Charly Caredda ${ }^{a, *}$, Laurent Mahieu-Williame $^{a}$, Raphaël Sablong ${ }^{a}$, Michaël Sdika $^{a}$, \\ Fabien C. Schneider ${ }^{b}$, Jacques Guyotat ${ }^{c}$ and Bruno Montcel ${ }^{a, *}$ \\ ${ }^{a}$ Université de Lyon, INSA-Lyon, Université Claude Bernard Lyon 1, UJM-Saint Etienne, CNRS, Inserm, \\ CREATIS UMR 5220, U1206, F69100 Lyon, France \\ ${ }^{b}$ Service de Radiologie, Centre Hospitalier Universitaire de Saint Etienne, TAPE EA7423, Université de Lyon, \\ UJM Saint Etienne, F42023 France \\ ${ }^{c}$ Service de Neurochirurgie D, Hospices Civils de Lyon, F69500 Bron, France \\ *charly.caredda@creatis.insa-lyon.fr,bruno.montcel@creatis.insa-lyon.fr
}

\begin{abstract}
We present the methodology for the intraoperative identification of resting state networks using RGB imaging. The results show a good correlation between the resting state and the brain areas identified by electrical brain stimulation. (C) 2021 The Author(s)
\end{abstract}

\section{Introduction}

Non invasive functional brain mapping is an imaging technique used to localize the functional areas of the patient brain. This technique is used during brain tumor resection surgery to indicate to the neurosurgeon the cortical tissues which should not be removed without cognitive impairment. Functional magnetic resonance imaging (fMRI) [1] is widely used to localize patient functional areas. However, after patient craniotomy, a brain shift invalidates the relevance of neuronavigation to intraoperatively localize the functional areas of the patient brain [2]. In order to prevent any localization error, intraoperative MRI has been suggested but it complicates the surgery gesture which makes it rarely used. For these reasons, Electrical brain stimulation (EBS) [3] is preferred during neurosurgery, but this technique is mainly limited by its low spatial resolution and has the potential risk to trigger epileptic seizures. Optical imaging provides an ideal solution for intraoperative functional brain mapping because the analysis of the light absorption allows to monitor the brain activity with quantification of the concentration changes in oxy- $\left(\Delta C_{\mathrm{HbO}_{2}}\right)$ and deoxygenated hemoglobin $\left(\Delta C_{H b}\right)$ in brain cortex [4].

As opposed to functional task-based analyses, resting state functional connectivity aims to identify the low frequency cerebral hemodynamic fluctuations $(<0.1 \mathrm{~Hz})$ that reflect the patient neuronal activity at rest and that are linked to resting state networks [5]. These fluctuations can occur in the absence of a task, and are often correlated between functionally related areas. Resting state functional connectivity is an emerging technique of public health because it allows the identification of functional brain areas without patient intervention and can potentially be used under general anesthesia.

In these works we demonstrate that optical resting state can be implemented intraoperatively using the seed correlation and independent component analysis methods and that we retrieve the motor brain areas identified by the electrical brain stimulation and optical task-based analysis. These results could have the potential to reduce the duration of surgical operations while improving patient and neurosurgeon comfort.

\section{Material and methods}

\subsection{Experimental Setup}

The wide field optical device employed in this study is presented in Fig. 1 and described in Ref [4]. This device is composed of a RGB camera (BASLER acA2000-165uc) in conjunction with an Edmund Optics camera lens $(f=50 \mathrm{~mm} f / 2-f / 22)$, a continuous wave white light source (OSRAM Classic 116W 230V light bulb) and a laptop (processor: Intel Core $15-7200 U, 2.50 \mathrm{GHz} \times 4$, ram: $15.3 \mathrm{GiB}$ ). The camera provided 8 bits RGB images every $33 m s$. 


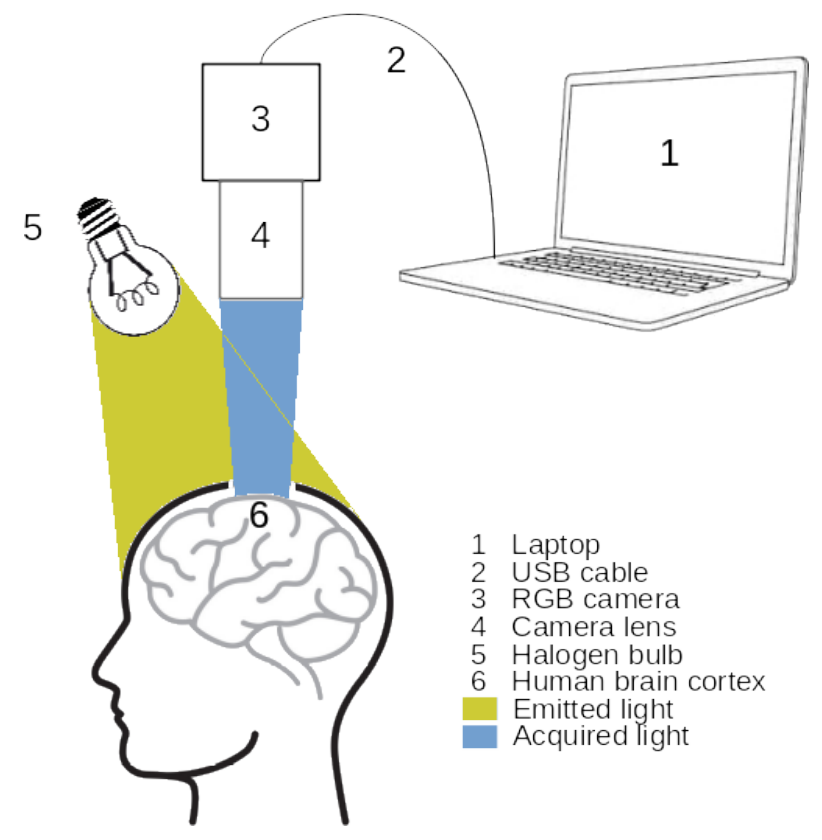

Fig. 1. Schematic of the imaging system.

\subsection{Patient Inclusion and Experimental Paradigm}

The study was conducted at the neurologic center of the Pierre Wertheimer hospital in Bron, France. A patient presenting a tumor close to the motor cortex area was included in the study and was under general anesthesia. Two different videos were acquired after patient craniotomy and before brain tumor resection operation: a 2 min acquisition during patient rest and a 2 min acquisition during a functional stimulation of the patient motor cortex. The stimulation of the cortex was achieved by two 20s periods of repetitive right fist clenching separated by rest periods (performed by an external person). After the acquisitions, the neurosurgeon operated EBS to localize the patient's brain motor area.

\subsection{Functional analyses}

Once the two videos were acquired, the quantitative model described in Ref [4] was applied to obtain $\Delta C_{H b}$ and $\Delta C_{\mathrm{HbO}_{2}}$ time courses for each camera pixel. For the video acquired with the patient at rest, two resting state analyses were computed to identify the patient's brain motor area: the seed correlation [6] and the independent components analyses (ICA) [7]. For the video acquired during a functional stimulation of the patient motor cortex, the patient's brain motor area was identified by calculating the Pearson correlation coefficient between the concentration time courses and the expected hemodynamic response [4]. The resting state and task-based results were compared to the EBS identification. The resting state analyses were compared to the task-based analysis using the Pearson correlation coefficient.

\section{Results and discussion}

In Fig. 2, the first image of the video sequence is represented in image (a). In image (b), the Pearson correlation coefficient computed between the $\Delta C_{H b}$ time courses and the expected hemodynamic response are plotted (taskbased analysis). In image (c), the Pearson correlation coefficient computed between the $\Delta C_{H b}$ time courses and those computed at the level of the seed are represented. In image (d), the normalized spatial distribution of $\Delta C_{H b}$ fluctuations identified by the ICA analysis are plotted. The spatial patterns (high values) in images (b), (c) and (d) correspond to the motor area identified by EBS (letter $\mathbf{M}$ ). The images obtained with the resting state analyses correspond to the image obtained with the task-based analysis. The Pearson correlation coefficient computed between images (b) and (c) is 0.47 and the one computed between images (b) and (d) is 0.46 . 


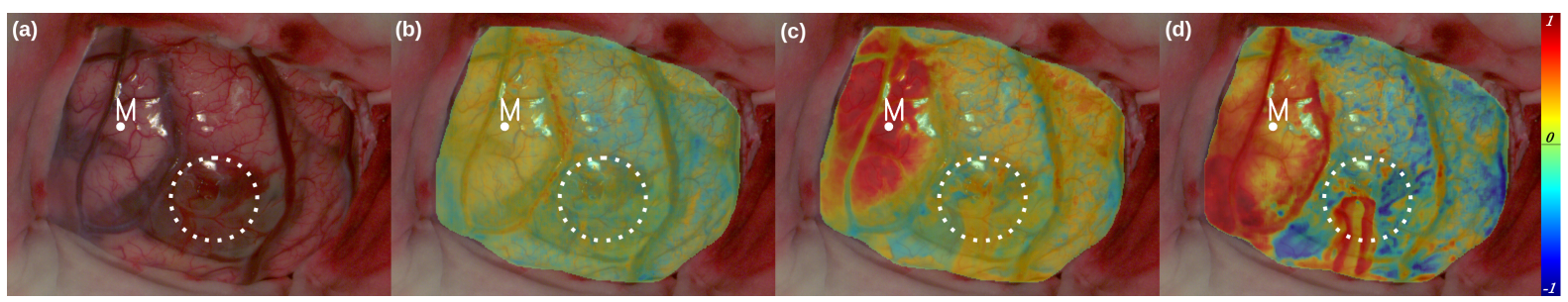

Fig. 2. (a) First image of the video sequence. (b) $\Delta C_{H b}$ task-based analysis. (c) $\Delta C_{H b}$ seed-based resting state analysis (the seed is indicated by the white spot). (d) Spatial distribution of $\Delta C_{H b}$ fluctuations identified by the ICA resting state analysis. In these images, the white spot and the letter $\mathbf{M}$ indicate the motor area identified by electrical brain stimulation. The dotted white circle delimits the patient's tumor. The colorbar represents the range of variation of the Pearson correlation coefficient in images (b) and (c) and the normalized spatial $\Delta C_{H b}$ fluctuations in image (d).

\section{Conclusion}

In this study, we present the methodology for the identification of resting state networks using RGB imaging during neurosurgery. The detection of functional brain areas using the resting state seed and ICA analyses present a strong similarity with the ones identified with a task-based analysis. These areas also correspond to the identification provided by electrical brain stimulation. The current work demonstrates that an RGB camera combined with a quantitative modeling of brain hemodynamics biomarkers could evaluate in a robust way the functional areas during patient rest and before a brain tumor resection operation. This strengthens the relevance of using a classical RGB camera for functional intraoperative brain imaging.

\section{Acknowledgments}

These works were funded by LABEX PRIMES (ANR-11-LABX-0063) of Université de Lyon, within the program "Investissements d'Avenir" (ANR-11-IDEX-0007), operated by the French National Research Agency (ANR); Cancéropôle Lyon Auvergne Rhône Alpes (CLARA) within the program “OncoStarter"; Infrastructures d'Avenir en Biologie Santé (ANR-11-INBS-000), within the program "Investissements d'Avenir" operated by the French National Research Agency (ANR) and France Life Imaging (ANR-11-INBS-0006). We want to acknowledge the PILoT facility for the support provided for the image acquisition.

\section{References}

1. S. Ogawa et al. Brain magnetic resonance imaging with contrast dependent on blood oxygenation. Proceedings of the National Academy of Sciences, 87(24):9868-9872, December 1990.

2. Ian J. Gerard et al. Brain shift in neuronavigation of brain tumors: A review. Medical Image Analysis, 35:403-420, January 2017.

3. Wilder Penfield et al. Somatic motor and sensory representation in the cerebral cortex of man as studied by electrical stimulation. Brain, 60(4):389-443, 1937.

4. Charly Caredda et al. Intraoperative quantitative functional brain mapping using an RGB camera. Neurophotonics, 6(4):1 - 14, 2019.

5. Michael D. Fox et al. Spontaneous fluctuations in brain activity observed with functional magnetic resonance imaging. Nature Reviews Neuroscience, 8(9):700-711, September 2007.

6. Rickson C. Mesquita et al. Resting state functional connectivity of the whole head with near-infrared spectroscopy. Biomedical Optics Express, 1(1):324, August 2010.

7. Brian R. White et al. Bedside optical imaging of occipital resting-state functional connectivity in neonates. NeuroImage, 59(3):2529-2538, February 2012. 